\title{
Majorization Algorithms for Inspecting Circles, Ellipses, Squares, Rectangles, and Rhombi
}

\author{
K. Van Deun \\ Department of Psychology, Catholic University of Leuven, \\ Tiensestraat 102, B-3000 Leuven, Belgium, katrijn.vandeun@psy.kuleuven.ac.be

\section{P. J. F. Groenen} \\ Econometric Instititute, Erasmus University Rotterdam \\ P.O. Box 1738, 3000 DR Rotterdam, The Netherlands, groenen@few.eur.nl
}

Econometric Institute Report EI 2003-35

\begin{abstract}
In several disciplines, as diverse as shape analysis, location theory, quality control, archaeology, and psychometrics, it can be of interest to fit a circle through a set of points. We use the result that it suffices to locate a center for which the variance of the distances from the center to a set of given points is minimal. In this paper, we propose a new algorithm based on iterative majorization to locate the center. This algorithm is guaranteed to yield a series nonincreasing variances until a stationary point is obtained. In all practical cases, the stationary point turns out to be a local minimum. Numerical experiments show that the majorizing algorithm is stable and fast. In addition, we extend the method to fit other shapes, such as a square, an ellipse, a rectangle, and a rhombus by making use of the class of $l_{p}$ distances and dimension weighting. In addition, we allow for rotations for shapes that might be rotated in the plane. We illustrate how this extended algorithm can be used as a tool for shape recognition.
\end{abstract}

Subject Classifications: Mathematics: functions: majorizing functions. Facilities: location: continuous. Engineering: shape analysis. 


\section{Introduction}

The central problem of this paper can be described in a simple manner: given a set of points, what is the best circle through these points. This task is equivalent to finding a center point such that its distance to all of given points is equal. It turns out that this problem appears in several disciplines. For example, in location analysis, this objective is known as the balancing or equity objective (Eiselt and Laporte 1990) and is often applied to decisions where the public needs to be served in a fair manner. An example is finding a location for a new library such that it is located at more or less the same distance for all its users. In the domain of pattern recognition, this criterion is known as least squares orthogonal distance fitting, see, for example, Ahn, Rauh, and Warnecke (2001) with applications in image processing. The problem of finding a circle that comes closest to a set of points forms a special case, with applications for example in archeology (Rorres and Romano 1997), computerized roundness inspection (Van-Ban and Lee 1991, Yeralan and Ventura 1988) and unfolding (Van Deun et al. in press).

Several algorithms have been proposed that solve the minimum variance problem. Many of these algorithms are computationally slow and strongly depend on the starting position (Ahn, Rauh, and Warnecke 2001). Often they are developed for a specific shape in a certain dimensionality usually chosen to be two or three. In this paper, we will show how the numerical optimization technique of iterative majorization can be used as a robust and computationally inexpensive tool to fit different shapes. Here, we will consider the two-dimensional shapes of circles, ellipses, squares, rectangles, and rhombi that are possibly rotated. Even though this paper is limited to two-dimensional shapes, the proposed algorithms can be used for more than two dimensions as well.

Consider the situation that a circle needs to be fitted through a given set of points where the coordinates of $n$ points are given by the rows of the $n \times 2$ matrix $\mathbf{Z}$ and row $i$ is given by the row vector $\mathbf{z}_{i}^{\prime}$. Throughout this paper, we shall denote vectors by boldface lowercase roman letters and matrices by boldface uppercase roman letters. If all $\mathbf{z}_{i}$ lie exactly on a circle, then there exists a center point $\mathbf{x}$ and a radius $R$ such that all the distances $d_{i}$ between $\mathbf{x}$ and $\mathbf{z}_{i}$ equals $R$. Therefore, the minimization of

$$
L(\mathbf{x}, R)=\sum_{i=1}^{n}\left(d_{i}-R\right)^{2},
$$

has a global minimum of zero whenever the points $\mathbf{z}_{i}$ fit exactly on a circle. If the points are almost on a circle, then (1) can be used as a least-squares 


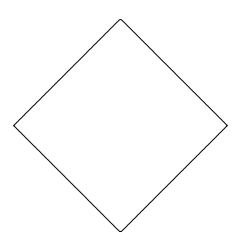

(a) $l_{1}$ ball

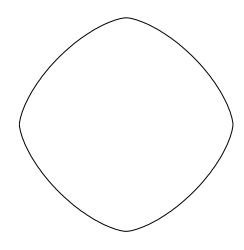

(b) $l_{3 / 2}$ ball

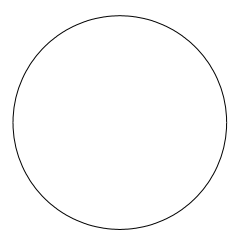

(c) $l_{2}$ ball

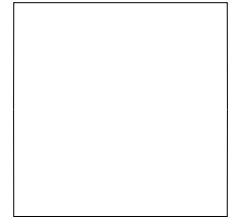

(d) $l_{\infty}$ ball

Figure 1: Contour line for points with unity distance to the origin. The different panels refer to different $l_{p}$ metrics.

model to fit a circle. Note that the size of the circle is not important as it is modelled by the radius $R$.

To fit a circle, $d_{i}$ needs to be defined as the Euclidean distance. Here, we propose to use its generalization, the Minkowski or $l_{p}$-distance

$$
d_{i}=\left(\sum_{s=1}^{m}\left|u_{i s}\right|^{p}\right)^{1 / p},
$$

with $p \geq 1$ the so called Minkowski parameter, $m$ the dimensionality (throughout this paper $m=2$ ), and

$$
u_{i s}=x_{s}-z_{i s} .
$$

Special cases of the $l_{p}$-distance are the Euclidean distance for $p=2$, the rectangular or city-block distance for $p=1$, and the infinity norm or dominance distance for $p=\infty$. The different shapes that can be fitted by (1) for different choices of $p$ are given in Figure 1. The shape corresponding to the Euclidean distance $\left(l_{2}\right)$ is the well known circle, for the rectangular distance $\left(l_{1}\right)$ a diamond (in two dimensions a rotated square), and for the dominance distance $\left(l_{\infty}\right)$ a square. For example, minimizing (1) for $p=1$ implies searching for the best fitting diamond.

Drezner, Steiner, and Wesolowsky (2002) showed that minimizing (1) is equivalent to minimizing,

$$
\begin{aligned}
L(\mathbf{x}) & =\sum_{i=1}^{n}\left(d_{i}-\frac{1}{n} \sum_{i} d_{i}\right)^{2} \\
& =\sum_{i=1}^{n} d_{i}^{2}-\frac{1}{n}\left(\sum_{i=1}^{n} d_{i}\right)^{2}
\end{aligned}
$$


Table 1: Coordinates for 9 points.

\begin{tabular}{l|ccccccccc}
\hline & $\mathbf{z}_{1}$ & $\mathbf{z}_{2}$ & $\mathbf{z}_{3}$ & $\mathbf{z}_{4}$ & $\mathbf{z}_{5}$ & $\mathbf{z}_{6}$ & $\mathbf{z}_{7}$ & $\mathbf{z}_{8}$ & $\mathbf{z}_{9}$ \\
\hline First dimension & -9 & -11 & 2 & -1 & 4 & 9 & 7 & 7 & 10 \\
Second dimension & 2 & -1 & 10 & -10 & 9 & -5 & 7 & -7 & 1 \\
\hline
\end{tabular}

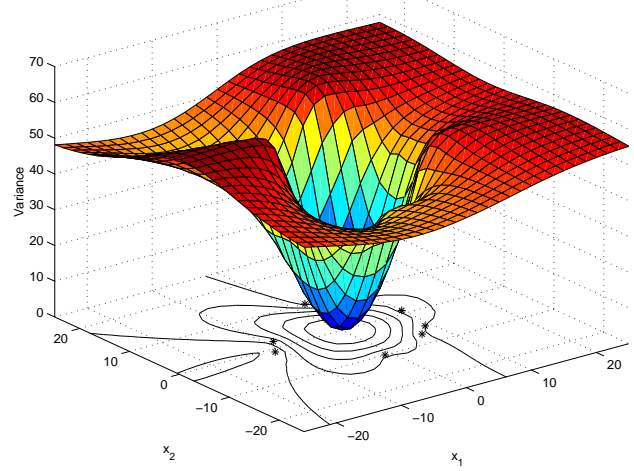

Figure 2: Variance in function of the center coordinates. The stars indicate the given points $\mathbf{z}_{i}$

which is equal to the variance of the distances if $L(\mathbf{x})$ is divided by $n$. The advantage of (4) over (1) is that we do not longer need to estimate the radius but only the coordinates of the center. In addition, (4) allows us to graphically display $L(\mathbf{x})$ as a function of the two center coordinates. Consider the data reported by Drezner, Steiner, and Wesolowsky (2002) of nine points that lie approximately on a circle (see Table 1). Figure 2 shows the value of the loss function as function of the center coordinates $\mathbf{x}$. Note that the nine points are also plotted as stars in the horizontal plane. In Figure 2, it is easy to see that $L(\mathbf{x})$ is not necessarily convex and has a steep decline in the region bounded by the given points.

This paper is organized as follows. First, we will develop an iterative majorization algorithm that can be used to find the point with minimum variance of the distances to a fixed set of points. Then, the algorithm is applied to the problem of finding the circle that comes closest to a set of points and we compare it with a Newton-Rhapson approach developed by Drezner, Steiner, and Wesolowsky (2002). Finally, we will show how our algorithm can be extended to model different shapes, such as squares, ellipses, and rectangles. For these shapes, we also allow for rotations. As a possible application, we consider the problem of shape recognition. 


\section{Minimizing $L(\mathrm{x})$ by Iterative Majorization}

To solve the problem of finding coordinates for the center that minimize (1) or, equivalently, (4) an iterative majorization procedure is developed. First, a general description of iterative majorization is presented. Then, we will show how a majorizing function that is quadratic in the coordinates can be obtained.

\subsection{Iterative Majorization}

Iterative majorization is a guaranteed descent method that has been mostly developed in the area of psychometrics and statistics. For a general introduction to iterative majorization, we refer to De Leeuw (1994), Heiser (1995), Lange, Hunter, and Yang (2000), and Kiers (2002). Here, we give a short description. The main idea is to replace in each iteration the objective function by an auxiliary function (called the majorizing function) that is easier to minimize such as a quadratic function. Then, an update of the majorizing function is easy to find. By choosing the majorizing function in a special manner, the important property of iterative majorization can be proven, that is, the property that the function value will never increase in subsequent iterations. In other words, for almost all practical applications, iterative majorization guarantees that the function values decrease. If the original function is bounded below by zero, as (4), the sequence of function values converges.

Let the majorizing function be denoted by $g\left(\mathbf{x}, \mathbf{x}_{0}\right)$, where $\mathbf{x}_{0}$ is called the supporting point which is the current known estimate of $\mathbf{x}$. The majorizing function has to have the following properties:

1. The majorizing function $g\left(\mathbf{x}, \mathbf{x}_{0}\right)$ should be easier to minimize than the original function $f(\mathbf{x})$.

2. The majorizing function is larger than or equal to the original function, that is, $f(\mathbf{x}) \leq g\left(\mathbf{x}, \mathbf{x}_{0}\right)$ for all $\mathbf{x}$.

3. The majorizing function touches the original function at the supporting point $\mathbf{x}_{0}$, that is, $g\left(\mathbf{x}_{0}, \mathbf{x}_{0}\right)=f\left(\mathbf{x}_{0}\right)$.

Let $\mathbf{x}^{+}$be the minimum of $g\left(\mathbf{x}, \mathbf{x}_{0}\right)$. From the two last properties it follows that

$$
f\left(\mathbf{x}^{+}\right) \leq g\left(\mathbf{x}^{+}, \mathbf{x}_{0}\right) \leq g\left(\mathbf{x}_{0}, \mathbf{x}_{0}\right)=f\left(\mathbf{x}_{0}\right)
$$

which is called the sandwich inequality by De Leeuw (1994). By choosing $\mathbf{x}_{0}$ of the current iteration as $\mathbf{x}^{+}$from the previous iteration, it follows that 
a majorizing algorithm yields a nonincreasing sequence of function values. Even though it can only be guaranteed that the algorithm will stop at a stationary point (provided that the function is differentiable), the guaranteed descent property is in almost all practical applications sufficient to stop at a local minimum.

Generally, the difficult part of iterative majorization lies in finding the majorizing function. However, one can use the overview of building blocks for majorizing functions given by Kiers (2002) and Groenen (2002).

\subsection{Obtaining a Majorizing Function for $L(\mathrm{x})$}

We now develop a majorizing function for $L(\mathbf{x})$. We use the fact that majorizing functions can be majorized themselves as well and the algorithm remains convergent. Below, we develop the majorizing function in steps. First, we majorize $L(\mathbf{x})$ to obtain a function that is either quadratic in $d_{i}$ or is a sum of $-d_{i}$. Then, we majorize $d_{i}^{2}$ and $-d_{i}$. Finally, these results are combined to obtain a quadratic function in $\mathbf{x}$.

The first term of $L(\mathbf{x})$ consists of a sum of $d_{i}^{2}$. The second term of $L(\mathbf{x})$, $-n^{-1}\left(\sum_{i=1}^{n} d_{i}\right)^{2}$, can be seen as $n^{-1}$ times minus the square of $\left(\sum_{i=1}^{n} d_{i}\right)$. Developing the inequality $c\left(t-t_{0}\right)^{2} \geq 0$ gives

$$
\begin{aligned}
c\left(t^{2}+t_{0}^{2}-2 t t_{0}\right) & \geq 0 \\
-c t^{2} & \leq-2 c t t_{0}+c t_{0}^{2}
\end{aligned}
$$

where $c$ is a positive value. Substituting $t=\sum_{i} d_{i}$ and $t_{0}=\sum_{i} d_{i 0}$ gives

$$
L(\mathbf{x}) \leq \sum_{i=1}^{n} d_{i}^{2}-\frac{2}{n}\left(\sum_{i=1}^{n} d_{i}\right)\left(\sum_{i=1}^{n} d_{i 0}\right)+k_{1},
$$

with $d_{i 0}$ the distances based on $\mathbf{x}_{0}$ (the previous estimation of $\mathbf{x}$ ) and $k_{1}=$ $n^{-1}\left(\sum_{i} d_{i 0}\right)^{2}$. Thus, the right hand part of (6) gives a majorizing function that consists of squared distances and a weighted sum of minus the distances.

We now focus on majorizing $-d_{i}$. Making use of Hölder's inequality, Groenen, Heiser, and Meulman (1999) found a majorizing function for $-d_{i}$ given as

$$
-d_{i} \leq-\sum_{s=1}^{m} u_{i s} u_{i s 0} b_{i s}^{(1)}
$$


where $u_{i s 0}$ is defined by (3) using the previous estimate $\mathbf{x}_{0}$ instead of $\mathbf{x}$ and

$$
b_{i s}^{(1)}= \begin{cases}\left|u_{i s 0}\right|^{p-2} / d_{i 0}^{p-1} & \text { if }\left|u_{i s 0}\right|>0 \text { and } 1 \leq p<\infty \\ 0 & \text { if }\left|u_{i s 0}\right|=0 \text { and } 1 \leq p<\infty \\ \left|u_{i s 0}\right|^{-1} & \text { if }\left|u_{i s 0}\right|>0, p=\infty, \text { and } s=s^{*} \\ 0 & \text { if }\left(\left|u_{i s 0}\right|=0 \text { or } s \neq s^{*}\right) \text { and } p=\infty\end{cases}
$$

where $s^{*}$ is defined for $p=\infty$ as the dimension on which the distance is maximal, i.e., $d_{i}=\left|u_{i s^{*}}\right|$.

For $d_{i}^{2}$ Groenen, Heiser, and Meulman (1999) also gave a quadratic majorizing function by

$$
d_{i}^{2} \leq \sum_{s=1}^{m} a_{i s} u_{i s}^{2}-2 \sum_{s=1}^{m} u_{i s} u_{i s 0} b_{i s}^{(2)}+\sum_{s=1}^{m} c_{i s}
$$

where

$$
a_{i s}= \begin{cases}\left|u_{i s 0}\right|^{p-2} / d_{i 0}^{p-2} & \text { if }\left|u_{i s 0}\right|>\epsilon \text { and } 1 \leq p \leq 2, \\ |\epsilon|^{p-2} / d_{i 0}^{p-2} & \text { if }\left|u_{i s 0}\right| \leq \epsilon \text { and } 1 \leq p \leq 2, \\ 2(p-1) & 2<p<\infty \\ \left|u_{i \phi_{1} 0}\right| /\left(\left|u_{i \phi_{1} 0}\right|-\left|u_{i \phi_{2} 0}\right|\right) & \text { if }\left|u_{i \phi_{1} 0}\right|-\left|u_{i \phi_{2} 0}\right|>\epsilon \text { and } p=\infty \\ \left(\left|u_{i \phi_{1} 0}\right|+\epsilon\right) / \epsilon & \text { if }\left|u_{i \phi_{1} 0}\right|-\left|u_{i \phi_{2} 0}\right| \leq \epsilon \text { and } p=\infty\end{cases}
$$

with $\phi_{s}$ an index that orders the values of $\left|u_{i s 0}\right|$ decreasingly (thus $\left|u_{i \phi_{1} 0}\right| \geq$ $\left.\left|u_{i \phi_{2} 0}\right|\right)$,

$$
b_{i s}^{(2)}= \begin{cases}0 & \text { if } 1 \leq p \leq 2 \\ a_{i s}-\left|u_{i s 0}\right|^{p-2} / d_{i 0}^{p-2} & \text { if }\left|u_{i s 0}\right|>0 \text { and } 2<p<\infty \\ 0 & \text { if }\left|u_{i s 0}\right|=0 \text { and } 2<p<\infty \\ a_{i s} & \text { if } s \neq \phi_{1} \text { and } p=\infty \\ a_{i s}\left|u_{i \phi_{2} 0}\right| /\left|u_{i \phi_{1} 0}\right| & \text { if } s=\phi_{1}, p=\infty\end{cases}
$$

and

$$
c_{i s}= \begin{cases}0 & \text { if } 1 \leq p \leq 2, \\ \sum_{s} a_{i s} u_{i s 0}^{2}-d_{i 0}^{2} & \text { if } 2<p<\infty, \\ \sum_{s}\left(2 b_{i s}^{(2)}-a_{i s}\right) u_{i s 0}^{2}+d_{i 0}^{2} & \text { if } p=\infty .\end{cases}
$$

For the proof that (7) and (9) are indeed majorizing functions, we refer to Groenen, Heiser, and Meulman (1999). Note that if $\left|u_{i s 0}\right|=0$ for all $s$, then $d_{i 0}=0$ so that $d_{i 0}^{p-2}$ appearing in $a_{i s}$ is not defined. In this case, we simply replace $\left|u_{i s 0}\right|=\epsilon$ for some small $\epsilon$ so that $d_{i 0}>\epsilon$ in $a_{i s}$. Strictly 
speaking this adaptation violates the majorization requirements. However, by choosing $\epsilon$ sufficiently small the majorizing algorithm remains convergent in practice.

Applying the majorizing inequalities of (7) and (9) into the right hand part of (6) gives

$$
\begin{aligned}
L(\mathbf{x}) & \leq \sum_{i=1}^{n} d_{i}^{2}-\frac{2}{n}\left(\sum_{i=1}^{n} d_{i}\right)\left(\sum_{i=1}^{n} d_{i 0}\right)+k_{1} \\
& \leq \sum_{i s} a_{i s} u_{i s}^{2}-2 \sum_{i s} u_{i s} b_{i s}+k_{2},
\end{aligned}
$$

where $b_{i s}=\left[\left(n^{-1} \sum_{i} d_{i 0}\right) b_{i s}^{(1)}+b_{i s}^{(2)}\right] u_{i s 0}$ and $k_{2}=k_{1}+\sum_{i s} c_{i s}$. Inserting $x_{s}-z_{i s}$ for $u_{i s}$ in the right hand part of (13) gives

$$
\begin{aligned}
L(\mathbf{x}) & \leq g_{1}\left(\mathbf{x}, \mathbf{x}_{0}\right) \\
& =\sum_{i s} a_{i s}\left(x_{s}-z_{i s}\right)^{2}-2 \sum_{i s}\left(x_{s}-z_{i s}\right) b_{i s}+k_{2} \\
& =\sum_{s} x_{s}^{2} \sum_{i} a_{i s}-2 \sum_{s} x_{s} \sum_{i}\left(a_{i s} z_{i s}+b_{i s}\right)+k_{3},
\end{aligned}
$$

where $k_{3}=k_{2}+\sum_{i s} a_{i s} z_{i s}^{2}+2 \sum_{i s} z_{i s} b_{i s}$. Inspecting $g_{1}\left(\mathbf{x}, \mathbf{x}_{0}\right)$ shows that the majorizing function in the right hand part of (14) is indeed quadratic in $x_{s}$. The minimum of a quadratic function can easily be obtained by setting the derivative equal to zero and solving for $x_{s}$, giving the update

$$
x_{s}^{+}=\frac{\sum_{i}\left(a_{i s} z_{i s}+b_{i s}\right)}{\sum_{i} a_{i s}} .
$$

The iterative majorization algorithm is given by

1. Choose initial $\mathbf{x}^{(0)}$. Set iteration counter $l:=0$ and set the convergence criterion $\epsilon_{\mathrm{conv}}$ to a small positive constant.

2. $l:=l+1$.

3. Calculate the update $\mathbf{x}^{+}$using (15) where $\mathbf{x}_{0}=\mathbf{x}^{(l-1)}$.

4. $\mathbf{x}^{(l+1)}=2 \mathbf{x}^{+}-\mathbf{x}^{(l-1)}$.

5. Check for convergence: If $\left\|\mathbf{x}^{(l-1)}-\mathbf{x}^{(l)}\right\|>\epsilon_{\text {conv }}$ go to Step 2 .

As a rational starting point for $\mathbf{x}^{(0)}$, we use the centroid of the data points, i.e., $\mathbf{x}^{(0)}=n^{-1} \sum_{i} \mathbf{z}_{i}$. 
Step 4 is an acceleration method that can be used with iterative majorization (De Leeuw and Heiser 1980, Heiser 1995). It can be proved that the use of the so-called relaxed update still guarantees a nonincreasing sequence of the variance of the distances (see Groenen, Mathar, and Heiser 1995) while experience shows that the number of iterations will be approximately halved.

\subsection{Minimizing the Variance of Euclidean Distances by Iter- ative Majorization}

For fitting circles, we have to use Euclidean distances. In this case, the update (15) of the majorizing algorithm simplifies considerably. In fact, for $p=2$,

$$
\begin{aligned}
a_{i s} & =1, \\
b_{i s}^{(1)} & = \begin{cases}d_{i 0}^{-1} & \text { if } d_{i 0}>0, \\
0 & \text { if } d_{i 0}=0\end{cases} \\
b_{i s}^{(2)} & =0 \\
c_{i s} & =0 \\
b_{i s} & =\left(n^{-1} \sum_{i} d_{i 0}\right) b_{i s}^{(1)} u_{i s 0}
\end{aligned}
$$

yielding the update

$$
x_{s}^{+}=n^{-1} \sum_{i}\left(z_{i s}+b_{i s}\right) .
$$

For Euclidean distances, the majorizing function is illustrated in Figure 3 where both the original loss function $L(\mathbf{x})$ and the majorizing function from the right hand side of (14) are plotted for the small data example of Table 1. Here the supporting point is chosen as $\mathbf{x}_{0}^{\prime}=\left[\begin{array}{ll}2 & 0\end{array}\right]$. As can be seen in Figure 3a, the majorizing function is a quadratic function that lies above the original loss function. In Figure $3 \mathrm{~b}$, we see that the majorizing function indeed touches the original function at the supporting point $\mathbf{x}_{0}$.

\section{Application: Finding the Best Fitting Circle}

We illustrate our algorithm by applying it to the problem of finding a circle that fits some given points. Several solutions have been proposed that minimize the variance of the distances of the center to the given points (see for example Ahn, Rauh, and Warnecke 2001, Drezner, Steiner, and Wesolowsky 


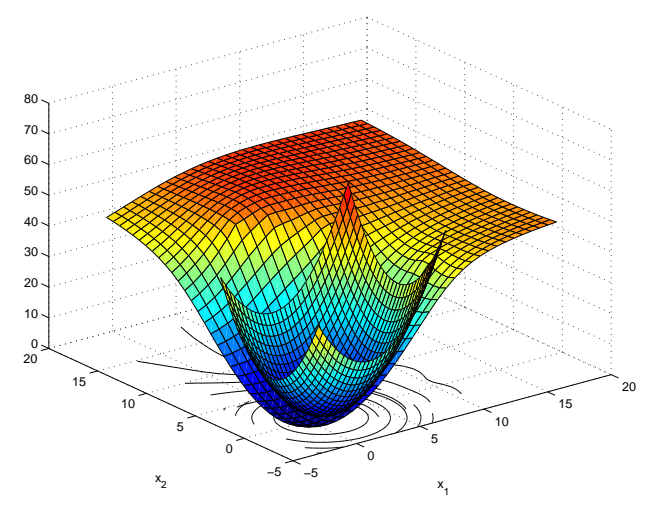

(a)

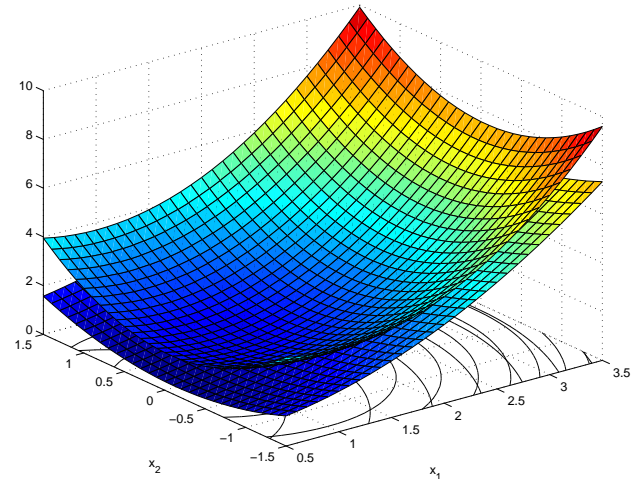

(b)

Figure 3: Plot of the original loss function and the quadratic majorizing function with supporting point $\mathbf{x}_{0}^{\prime}=\left[\begin{array}{ll}2 & 0\end{array}\right]$ (Panel a). Panel (b) shows a detail.

2002, Rorres and Romano 1997). Here, we limit our comparison to the iterative majorization algorithm and Newton-Raphson algorithm proposed by Drezner, Steiner, and Wesolowsky (2002). At the end of this section, we will further investigate the performance of the iterative majorization approach in a simulation study.

First, we compare the sensitivity of the two algorithms for the starting values of $\mathbf{x}^{(0)}$. Again, we use the nine points reported in Table 1. Both algorithms were stopped when the difference between the $L(\mathbf{x})$ values in two subsequent iterations was less than $10^{-5}$. This weaker convergence criterion was chosen for reasons given here below. To check the sensitivity for the start configuration, we started both algorithms from all points on a grid defined for $x_{1}$ and $x_{2}$ between $[-20,20]$ in steps of 2 . Then, the final $L(\mathbf{x})$ after convergence was noted. Figure 4 a shows these values on the $z$-axis for the Newton-Raphson algorithm of Drezner, Steiner, and Wesolowsky (2002) and for the majorization algorithm in Figure 4a. It can be seen clearly that the Newton-Raphson algorithm strongly depends on the starting position. Only for starting positions not too far from the center, the global minimum of $L(\mathbf{x})$ is found. Note that for some starting values, there is a gap in the figure. This gap is due to numerical problems which turn out to be more severe for stronger convergence criteria. The majorizing algorithm shows a flat surface indicating that from all starting positions with $-20 \leq x_{1} \leq 20$ 


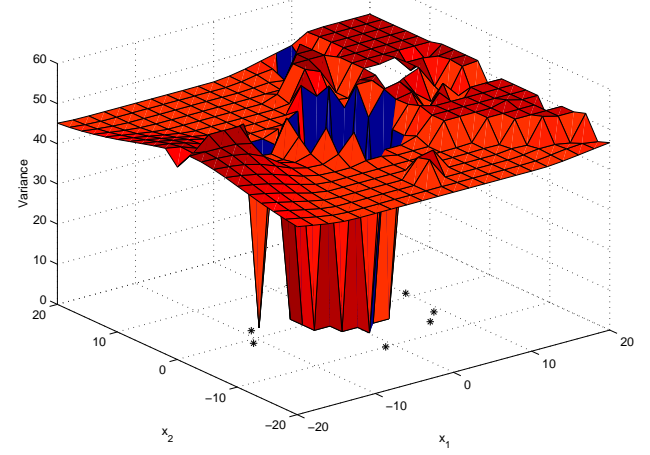

(a)

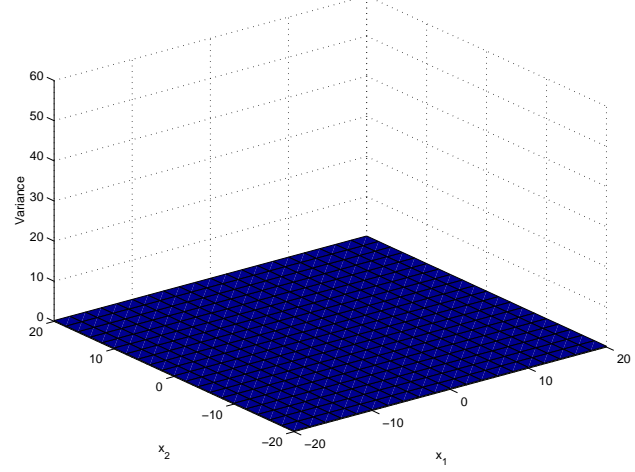

(b)

Figure 4: Value of the objective function for different starting positions. The left panel stems from the Newton Raphson approach and the right panel from the iterative majorization approach. The stars indicate the given points.

and $-20 \leq x_{2} \leq 20$, the global minimum of $L(\mathbf{x})$ is found. So even starting outside the circle leads to the global optimum.

Next, we compare both algorithms for starting positions close to the optimum. As a convergence criterion we take the distance between the updated center and the previous one: when this distance is smaller then $10^{-5}$, the iterative procedure is stopped. One thousand starting positions were generated uniformly on the disk centered around the optimum and with radius equal to one. The results are reported in Table 2. For each algorithm the minimum value of $L(\mathbf{x})$, the range of the $L(\mathbf{x})$ values, the minimum number of iterations, the maximum number of iterations and the total computing time are given. In terms of convergence, the Newton-Rhapson approach is faster as can be seen from the number of iterations and the range of the variance. However, in terms of CPU time the iterative majorization algorithm is about as fast.

We further investigate the performance of the iterative majorization approach in a small simulation study. In this study three factors are varied:

- the number of given points is varied $(n=3,4,6,10,20)$,

- the noise level ( $e=$ no noise, 5 percent), and

- the angle the given points cover on the circle $(a=90,180$, and 360 degrees). 
Table 2: Some measures of performance of the algorithms for the Drezner data. The first line shows the results for the Newton-Rhapson approach, the second line for the iterative majorization algorithm.

\begin{tabular}{lccccc}
\hline Algorithm & Min fit & Range fit & Min iter & Max iter & Time \\
\hline Newton-Raphson & 0.19883320109211 & $9.5 \mathrm{e}-16$ & 3 & 4 & 19.92 \\
Iterative Majorization & 0.19883320109211 & $6.4 \mathrm{e}-13$ & 4 & 7 & 20.12 \\
\hline
\end{tabular}
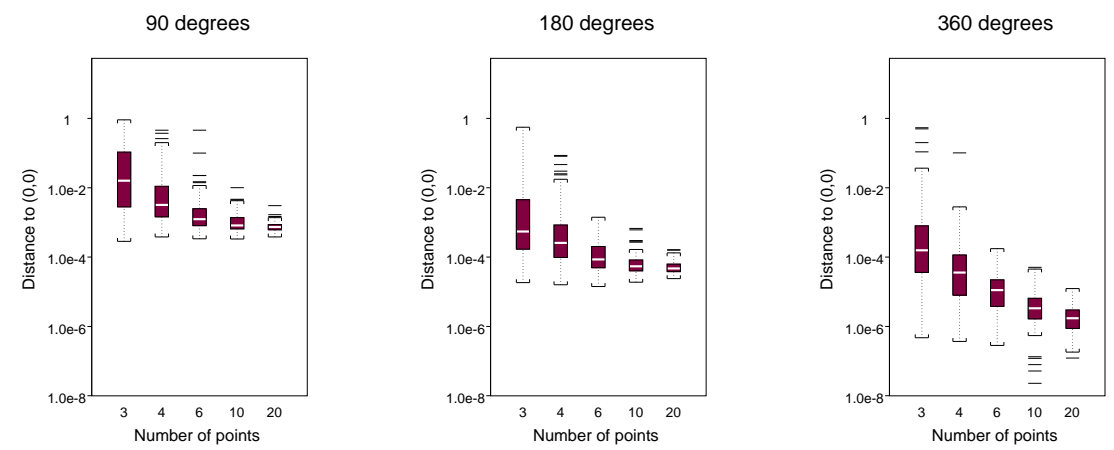

Figure 5: Boxplots of the distances between the true center and the center located by the algorithm in the simulation study for different $n$ and $a$ for error free data.

Per case, 100 times $\mathrm{n}$ points were generated on a part (covering $a$ degrees) of the circle with origin $(0,0)$ and radius one plus $e$. For each case, a starting point $\mathbf{x}^{(0)}$ was generated uniformly on the disc with radius one. The algorithms terminate using $\epsilon_{\mathrm{conv}}=10^{-5}$. In this study, the true center location was known a priori. Therefore, the success of the algorithm can be measured by the Euclidean distance between the center location found by the algorithm and the true origin at $(0,0)$. Figures 5 and 6 show the distribution of these distances by Boxplots for the various combinations of the factors in this study. Note that because the distributions are highly skew, a log scale is used in these Boxplots.

To investigate what effects are important we did an analysis of variance (ANOVA) using the log of the distances as the response variable, see Table 3 . The number of given points $n$ is treated as a random factor while both the coverage angle $a$ and the error level $e$ are treated as fixed factors. The effect size reflects the proportion of variance of the log distances that is accounted for by the effect. In total, 86 percent is accounted for. The main effects 

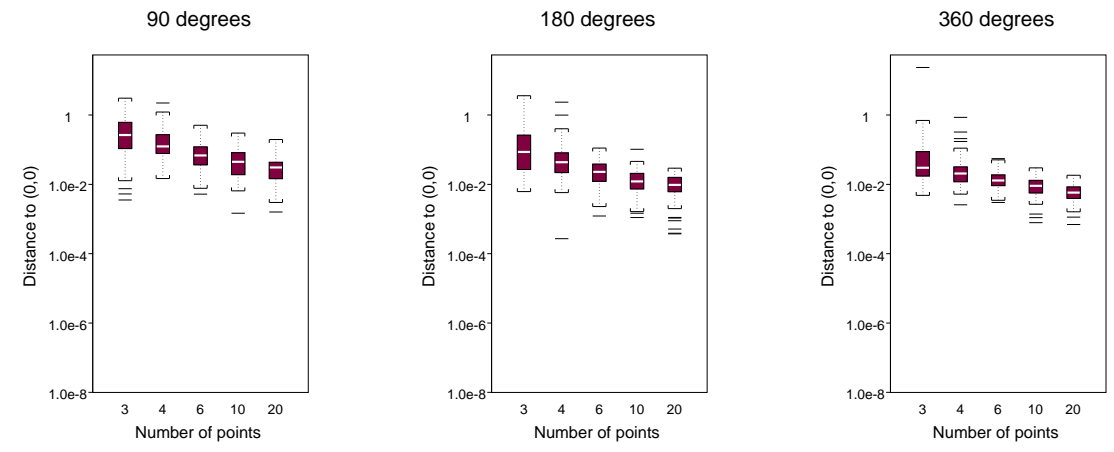

Figure 6: Boxplots of the distances between the true center and the center located by the algorithm in the simulation study for different $n$ and $a$ for error perturbed data.

Table 3: Analysis of variance table for log distances in the simulation study.

\begin{tabular}{lrrrrr}
\hline Source & SS & DF & MS & F & Effect size \\
\hline$e$ & 3712 & 1 & 3712 & 330.76 & 0.52 \\
$a$ & 1156 & 2 & 578 & 558.79 & 0.16 \\
$n$ & 654 & 4 & 163 & 502.00 & 0.11 \\
$e \times a$ & 311 & 2 & 155 & 68.63 & 0.04 \\
$e \times n$ & 45 & 4 & 11 & 34.43 & 0.02 \\
$a \times n$ & 8 & 8 & 1 & 3.17 & 0 \\
$e \times a \times n$ & 18 & 8 & 2 & 6.96 & 0 \\
Residual & 968 & 2970 & 0.33 & & \\
Total & 6873 & 2999 & & & \\
\hline
\end{tabular}

account for almost all explained variance, with 52 percent accounted for by the error level.

There is a large difference between the case with and without error: in absence of error, the solution lies clearly closer to the true origin. For the error free case (see Figure 5), both the angle and the number of points are influential. The solutions are clearly closer to the true solutions for a larger number of points and for larger coverage angles. This is less so for the case with error (Figure 6). 


\section{Extensions of Majorization}

So far we have seen how the best fitting circle through a number of given points can be found. The loss function we used is based on $l_{p}$ distances and we derived a majorizing function that is easy to minimize for $l_{p}$ distances with $p \geq 1$. This majorizing function was used to solve the minimum variance problem for Euclidean distances with as an application the problem of finding a circle. Minimizing the variance for other $l_{p}$ norms, allows us to fit different shapes such as the ones given in the first row of Figure 7. For example, taking $l_{1}$ or $l_{\infty}$ distances can be used to find the best fitting diamond or square respectively.

The loss function can be extended to include dimension weights and rotations. Dimension weights are used to differentially stretch the dimensions so that instead of a circle, diamond, or square we can fit an ellipse, rhombus, or rectangle (see the second row of Figure 7). The effect of rotations is illustrated in the third row of the same figure while the combined effect of dimension weighting and rotating results in the shapes in the bottom row of Figure 7. Note that the order of the transformations has to be respected, this is first apply dimension weights to the basic shapes of the top row in Figure 7 and then rotate to obtain the shapes in the bottom row.

In the remainder of this section, we will first introduce rotations and dimension weighting formally. Then we will illustrate how the algorithm can be used to solve the problem of shape recognition for a restricted class of shapes. An extension of the majorizing algorithm that searches for optimal center coordinates, dimension weights, and rotation is developed in the appendix.

\subsection{Rotations and Dimension Weighting}

Let $\mathbf{T}$ be a rotation matrix. Note that for any rotation matrix $\mathbf{T}$ is orthonormal, that is, $\mathbf{T}^{\prime} \mathbf{T}=\mathbf{T}^{-1} \mathbf{T}=\mathbf{I}$. In two dimensions, $\mathbf{T}$ can be written as

$$
\mathbf{T}=\left[\begin{array}{rr}
\cos \alpha & -\sin \alpha \\
\sin \alpha & \cos \alpha
\end{array}\right]
$$

where $\alpha$ is the rotation angle anticlockwise. Thus, ZT is an anticlockwise rotation of $\mathbf{Z}$ by $\alpha$ degrees. Choosing $\mathbf{T}$ as the identity matrix $\mathbf{I}$ leaves $\mathbf{Z T}=$ $\mathbf{Z I}=\mathbf{Z}$ unrotated. We also consider dimension weighting by a diagonal matrix $\mathbf{W}$, so that, in $\mathbf{Z W}$ the coordinates of $\mathbf{Z}$ along dimension $s$ are weighted by the factor $w_{s}$. Rotations and weighting can be combined by 


\begin{tabular}{ccccc}
\hline Rotation & $\begin{array}{c}\text { Dimension } \\
\text { weighting }\end{array}$ & $p=1$ & $p=1.5$ & $p=2$
\end{tabular}

Figure 7: Some shapes that can be fitted with $l_{p}$ distances for $p \geq 1$, allowing for dimension weighting and orthogonal rotations. Each column illustrates a certain $l_{p}$ norm. The rows show the shape that can be fitted, whether it is weighted (by $w_{1}=1$ and $\left.w_{2}=0.3\right)$ or unweighted $\left(w_{1}=1\right.$ and $\left.w_{2}=1\right)$ and rotated by 30 degrees or not.

$\mathbf{Z T W}$, which simply means first rotate $\mathbf{Z}$ by $\mathbf{T}$ and then weight the rotated axes by $\mathbf{W}$.

To adapt the variance function for different rotations and dimension weighting, we have to minimize

$$
L_{2}(\mathbf{x}, \mathbf{T}, \mathbf{W})=\sum_{i=1}^{n} d_{i}^{2}-\frac{1}{n}\left(\sum_{i=1}^{n} d_{i}\right)^{2}
$$

with $u_{i s}$ in (2) defined as

$$
u_{i s}=x_{s}-w_{s} \mathbf{z}_{i}^{\prime} \mathbf{t}_{s},
$$

where $\mathbf{t}_{s}$ is column $s$ of the rotation matrix $\mathbf{T}$. Here, the right order of operations is to first rotate and then weight the data. Only in this way we obtain a $l_{p}$ ball with minimum variance of the distances to the center. Some precaution is necessary, since minimizing $L_{2}(\mathbf{x}, \mathbf{T}, \mathbf{W})$ will trivially be zero when choosing $\mathbf{x}=\mathbf{0}$ and $\mathbf{W}=\mathbf{0}$. Therefore, we impose the 
additional restriction that $\operatorname{tr} \mathbf{W}^{2}=\sum_{s} w_{s}^{2}=m$ to avoid the degeneracy. As preliminary experimentation showed that occasionally solutions occurred with only one nonzero weight, we further impose the restriction that $w_{s} \geq$ $w_{\min }$ for all $s . w_{\min }$ is a small positive constant. In appendix we develop an iterative majorization algorithm that can be used to find $\mathbf{x}, \mathbf{T}$ and, $\mathbf{W}$ such that $L_{2}$ is minimized.

\subsection{Shape Recognition}

So far we considered the problem of fitting a predefined shape but the algorithm could also be used for shape recognition. For example, in reverse engineering (with applications in reproduction and redesign) the goal is to model engineering parts according to the original design (see for example Langbein et al. 2001) while in graphics recognition (see for example Fonseca and Jorge 2001) one wants to digitally recognize hand-drawn shapes. Apart from the problem that the model has to be noiseless, the shape has to be recognized under rotations and unknown scale. In the appendix, we developed an algorithm that can be used to solve this problem for a restricted class of shapes. Although we can fit any of the shapes in Figure 7 to our data (and all other $l_{p}$ balls with $p \geq 1$ ), we limit it to the case of the last row in Figure 7 (thus allowing for rotation and dimension weighting) for $p=1,2$, and $\infty$.

The proposed algorithm can be used to fit a weighted and rotated $l_{p}$ ball to the given points. By doing this for different $p$, the shape with the highest fit can be found. It is this shape that we will retain as the origin of the points. As a measure of fit we will consider a normalized variance. This is the variance of the $c d_{i}$ where $c$ is chosen such that the mean distance (and thus the radius $R$ ) equals one. In this way, the variances are comparable between different data sets. We give a small illustration for noiseless data.

Twelve points were generated on each of three different shapes: a diamond, an unrotated ellipsis (with weights $w_{1}=1.35$ and $w_{2}=0.41$ ) and a rotated ( $\alpha=30$ degrees anticlockwise) rectangle. We use the majorization algorithm given in appendix to find the center, weights, and rotation angle of the ball. In order to avoid local minima, 200 solutions were generated on the basis of random initial parameters. The numbers shown in Figure 8 are based on the solution with the lowest variance. For each shape, three rows are given with the estimated weights, rotation angle, and the scaled variance for one of the following norms: $l_{1}, l_{2}$ or $l_{\infty}$. The shapes are well detected as the original norm is recovered and as the parameter values approximate the underlying ones well. For example, in case of the ellipsis we find the 


\begin{tabular}{rrrrrr}
\hline Shape & norm & $\alpha$ & & $\mathbf{w}^{\prime}$ & Scaled Var \\
\hline & & & & & \\
& $l_{1}$ & 16 & {$[1.00$} & $1.00]$ & $1.0 \times 10^{-6}$ \\
$l_{2}$ & 58 & {$[1.05$} & $0.95]$ & $1.5 \times 10^{-2}$ \\
$l_{\infty}$ & 41 & {$[0.98$} & $1.02]$ & $7.3 \times 10^{-4}$ \\
& & & & & \\
$l_{1}$ & 164 & {$[1.37$} & $0.36]$ & $2.9 \times 10^{-3}$ \\
$l_{2}$ & 0 & {$[1.35$} & $0.41]$ & $9.9 \times 10^{-8}$ \\
& $l_{\infty}$ & 121 & {$[1.38$} & $0.32]$ & $7.6 \times 10^{-3}$ \\
& & & & \\
$l_{1}$ & 32 & {$[1.40$} & $0.22]$ & $4.0 \times 10^{-2}$ \\
$l_{2}$ & 29 & {$[1.37$} & $0.37]$ & $1.4 \times 10^{-2}$ \\
$l_{\infty}$ & 30 & {$[1.37$} & $0.41]$ & $3.6 \times 10^{-5}$ \\
& & & & & \\
\end{tabular}

Figure 8: Shape recognition. For each figure at the left, the rotation angle, the weights and the scaled variance obtained with the majorizing algorithm are reported. This is done for the $l_{1}, l_{2}$ and $l_{\infty}$ norm.

lowest variance for the Euclidean norm, the rotation angle equals zero and the weights equal $\left[\begin{array}{ll}1.35 & 0.41\end{array}\right]$. In this case, the true underlying values are fully recovered. In case of the diamond, the solution based on dominance distances fits well too with a rotation angle of almost 45 degrees. This comes as no surprise given that the diamond is a rotated (by 45 degrees) square.

\section{Conclusion and Discussion}

We showed how iterative majorization can be used to minimize the variance of distances which is a popular objective function in location theory and in pattern recognition. To be able to fit both circular and square shapes we extended the use of the Euclidean distance to $l_{p}$ distances including the cityblock distance and dominance distance. It was also shown how spherical, rectangular and, rhombic shapes could be fitted by including dimension weights in the loss function. In this case, too, iterative majorization was used to develop an algorithm to minimize the variance of the distances. A final extension is to include rotations so that the shapes may be rotated in space.

In a small numerical application on fitting circles, we showed that our algorithm is equally fast but more robust in comparison to a Newton-Raphson approach that was specifically developed to obtain a fast algorithm. In a different numerical experiment, we illustrated how our proposed algorithm 
can be used as a tool for shape recognition.

Here, we restricted ourselves to two-dimensional problems but the algorithms can be applied to higher dimensional problems without modification. In applications where some points may be measured (wrongly) to be far off the shape, it may be wise to robustify the variance function by changing to the mean absolute deviation as a loss criterion. In this case, a majorizing algorithm is possible as well, but it will be necessary to investigate how well such an algorithm is prone to local minima.

The algorithms in this paper can be applied to any number of given points $n>2$. It remains to be investigated how many points are mathematically necessary to detect a certain shape and how many points are needed in practice to guarantee a proper fit. These issues are to be studied in future research. 


\section{Appendix: A Majorizing Algorithm for $L_{2}(\mathbf{x}, \mathbf{T}, \mathbf{W})$}

The majorization derivations remain the same up to (13). Substituting the definition of $u_{i s}$ from (19) into (13) gives

$$
\begin{aligned}
& L_{2}(\mathbf{x}, \mathbf{T}, \mathbf{W}) \leq g_{2}(\mathbf{x}, \mathbf{T}, \mathbf{W}) \\
& \quad=\sum_{i s} a_{i s}\left(x_{s}-w_{s} \mathbf{z}_{i}^{\prime} \mathbf{t}_{s}\right)^{2}-2 \sum_{i s}\left(x_{s}-w_{s} \mathbf{z}_{i}^{\prime} \mathbf{t}_{s}\right) b_{i s}+k_{2} .
\end{aligned}
$$

Note that the majorizing function $g_{2}(\mathbf{x}, \mathbf{T}, \mathbf{W})$ is quadratic in $\mathbf{x}, \mathbf{T}$, and $\mathbf{W}$. We shall use alternating least squares to update the parameters, that is, $\mathbf{x}$ is updated while $\mathbf{T}$ and $\mathbf{W}$ are kept fixed, then $\mathbf{T}$ is updated while $\mathbf{x}$ and $\mathbf{W}$ are kept fixed, and finally $\mathbf{W}$ is updated while $\mathbf{x}$ and $\mathbf{T}$ are kept fixed.

Updating $\mathbf{x}$ is analogous to (15). $g_{2}(\mathbf{x}, \mathbf{T}, \mathbf{W})$ can be written as

$$
g_{2}(\mathbf{x}, \mathbf{T}, \mathbf{W})=\sum_{s} x_{s}^{2} \sum_{i} a_{i s}-2 \sum_{s} x_{s} \sum_{i}\left(a_{i s} w_{s} \mathbf{z}_{i}^{\prime} \mathbf{t}_{s}+b_{i s}\right)+k_{3},
$$

where $k_{3}$ is a constant not depending $\mathbf{x}$, i.e., $k_{3}=\sum_{i s} a_{i s} w_{s}^{2}\left(\mathbf{z}_{i}^{\prime} \mathbf{t}_{s}\right)^{2}+$ $2 \sum_{i s} w_{s} b_{i s} \mathbf{z}_{i}^{\prime} \mathbf{t}_{s}$. It can be shown that the optimal update minimizing $g_{2}(\mathbf{x}, \mathbf{T}, \mathbf{W})$ over $\mathbf{x}$ while keeping $\mathbf{T}$ and $\mathbf{W}$ fixed equals

$$
x_{s}^{+}=\frac{\sum_{i}\left(a_{i s} w_{s} \mathbf{z}_{i}^{\prime} \mathbf{t}_{s}+b_{i s}\right)}{\sum_{i} a_{i s}} .
$$

To update $\mathbf{W}$ for fixed $\mathbf{T}$ and $\mathbf{x}$, we write $g_{2}(\mathbf{x}, \mathbf{T}, \mathbf{W})$ as

$$
g_{2}(\mathbf{x}, \mathbf{T}, \mathbf{W})=\sum_{i s} a_{i s} w_{s}^{2}\left(\mathbf{z}_{i}^{\prime} \mathbf{t}_{s}\right)^{2}-2 \sum_{i s} w_{s} \mathbf{z}_{i}^{\prime} \mathbf{t}_{s}\left(x_{s} a_{i s}-b_{i s}\right)+k_{4},
$$

where $k_{4}$ is a constant not depending $\mathbf{W}$, i.e., $k_{4}=\sum_{i s} x_{s}^{2} a_{i s}-2 \sum_{i s} x_{s} b_{i s}$. Note that $\mathbf{W}$ has a length constraint such that $\sum_{s} w_{s}^{2}=m$. We first rewrite (23) as

$$
\begin{aligned}
g_{2} & (\mathbf{x}, \mathbf{T}, \mathbf{W}) \\
& =\sum_{s} w_{s}^{2} \sum_{i}\left[a_{i s}\left(\mathbf{z}_{i}^{\prime} \mathbf{t}_{s}\right)^{2}\right]-2 \sum_{s} w_{s}\left[\sum_{i} \mathbf{z}_{i}^{\prime} \mathbf{t}_{s}\left(x_{s} a_{i s}-b_{i s}\right)\right]+k_{4} \\
& =\sum_{s} w_{s}^{2} c_{s}-2 \sum_{s} w_{s} r_{s}+k_{5} \\
& =\mathbf{w}^{\prime} \mathbf{C}_{\mathbf{w}} \mathbf{w}-2 \mathbf{w}^{\prime} \mathbf{r}_{\mathbf{w}}+k_{4}
\end{aligned}
$$

where $\mathbf{w}$ is a vector with elements $w_{s}, \mathbf{C}_{\mathbf{w}}$ is a diagonal matrix with diagonal elements $c_{s}=a_{i s}\left(\mathbf{z}_{i}^{\prime} \mathbf{t}_{s}\right)^{2}$, and $\mathbf{r}_{\mathbf{w}}$ is a vector with elements $\sum_{i} \mathbf{z}_{i}^{\prime} \mathbf{t}_{s}\left(x_{s} a_{i s}-b_{i s}\right)$. 
Because $\mathbf{C}_{\mathbf{w}}$ is generally not equal to $\mathbf{I}$, the update $\mathbf{C}_{\mathbf{w}}^{-1} \mathbf{r}_{\mathbf{w}} /\left\|\mathbf{C}_{\mathbf{w}}^{-1} \mathbf{r}_{\mathbf{w}}\right\|$ is not the least squares update, because the metric $\mathbf{C}_{\mathbf{w}}$ in (24) is not equal to the metric used for the length constraint. We use an additional majorizing step to obtain a majorizing function that has $\mathbf{I}$ as a metric. Let $\lambda_{\mathbf{w}}$ be the largest eigenvalue of $\mathbf{C}_{\mathbf{w}}$. Then, the matrix $\mathbf{C}_{\mathbf{w}}-\lambda_{\mathbf{w}} \mathbf{I}$ is negative semi-definite, so that we obtain the inequality $\left(\mathbf{w}-\mathbf{w}_{0}\right)^{\prime}\left(\mathbf{C}_{\mathbf{w}}-\lambda_{\mathbf{w}} \mathbf{I}\right)\left(\mathbf{w}-\mathbf{w}_{0}\right) \leq 0$ with a strict equality if $\mathbf{w}=\mathbf{w}_{0}$. Expanding this inequality yields

$$
\begin{aligned}
\left(\mathbf{w}-\mathbf{w}_{0}\right)^{\prime} \mathbf{C}_{\mathbf{w}}\left(\mathbf{w}-\mathbf{w}_{0}\right) \leq & \lambda_{\mathbf{w}}\left(\mathbf{w}-\mathbf{w}_{0}\right)^{\prime}\left(\mathbf{w}-\mathbf{w}_{0}\right) \\
\mathbf{w}^{\prime} \mathbf{C}_{\mathbf{w}} \mathbf{w} \leq & \lambda_{\mathbf{w}} \mathbf{w}^{\prime} \mathbf{w}-2 \lambda_{\mathbf{w}} \mathbf{w}^{\prime}\left(\mathbf{w}_{0}-\lambda_{\mathbf{w}}^{-1} \mathbf{C} \mathbf{w}_{0}\right) \\
& +\mathbf{w}_{0}^{\prime}\left(\lambda_{\mathbf{w}} \mathbf{I}-\mathbf{C}_{\mathbf{w}}\right) \mathbf{w}_{0}
\end{aligned}
$$

so that

$$
\begin{aligned}
g_{2}(\mathbf{x}, \mathbf{T}, \mathbf{W}) \leq & \lambda_{\mathbf{w}} \mathbf{w}^{\prime} \mathbf{w}-2 \lambda_{\mathbf{w}} \mathbf{w}^{\prime}\left(\mathbf{w}_{0}+\lambda_{\mathbf{w}}^{-1}\left[\mathbf{r}_{\mathbf{w}}-\mathbf{C}_{\mathbf{w}} \mathbf{w}_{0}\right]\right) \\
& +\mathbf{w}_{0}^{\prime}\left(\lambda_{\mathbf{w}} \mathbf{I}-\mathbf{C}_{\mathbf{w}}\right) \mathbf{w}_{0}+k_{4} .
\end{aligned}
$$

In the right hand part of (26), the metric of the quadratic part in $\mathbf{w}$ does coincide with the metric of the length constraint, so that the minimum of (26) subject to the length-constraint is given by

$$
\mathbf{w}_{u}=m^{1 / 2} \mathbf{q} /\|\mathbf{q}\|
$$

where $\mathbf{q}=\mathbf{w}_{0}+\lambda_{\mathbf{w}}^{-1}\left[\mathbf{r}_{\mathbf{w}}-\mathbf{C}_{\mathbf{w}} \mathbf{w}_{0}\right]$. To impose the additional restriction that $w_{s} \geq w_{\min }$, the calculation of the weights should be adapted in the following way for the case that $m=2$ :

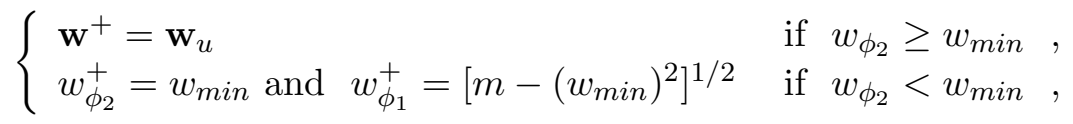

with $\phi_{s}$ an index that orders the values of $\mathbf{w}_{u}$ decreasingly. Note that $w_{\text {min }}$ must be (much) smaller than 1 for a solution to exist. If $m>2$, a different procedure is needed using an adaptation of the explicit solutions for lengthconstrained nonnegative least squares discussed in Groenen, van Os, and Meulman (2000).

Next, we wish to obtain an update for $\mathbf{T}$ for fixed $\mathbf{x}$ and $\mathbf{W}$. Note that $\mathbf{T}$ is constrained to be orthonormal. Within the frame work of iterative majorization, Kiers and Groenen (1996) showed that it is helpful to majorize quadratic functions in $\mathbf{T}$ in such a way that the metric is removed. This can be done by applying again the majorizing inequality of (25). Before we can 
do so, we rewrite $(23)$ as

$$
\begin{aligned}
g_{2} & (\mathbf{x}, \mathbf{T}, \mathbf{W}) \\
& =\sum_{s} \mathbf{t}_{s}^{\prime}\left[w_{s}^{2} \sum_{i} a_{i s} \mathbf{z}_{i} \mathbf{z}_{i}^{\prime}\right] \mathbf{t}_{s}-2 \sum_{s} \mathbf{t}_{s}^{\prime}\left[w_{s} \sum_{i} \mathbf{z}_{i}\left(x_{s} a_{i s}-b_{i s}\right)\right]+k_{4} \\
& =\sum_{s} \mathbf{t}_{s}^{\prime} \mathbf{C}_{\mathbf{t}_{s}} \mathbf{t}_{s}-2 \sum_{s} \mathbf{t}_{s}^{\prime} \mathbf{r}_{\mathbf{t}_{s}}+k_{4}
\end{aligned}
$$

where $\mathbf{C}_{\mathbf{t}_{s}}=w_{s}^{2} \sum_{i} a_{i s} \mathbf{z}_{i} \mathbf{z}_{i}^{\prime}$ and $\mathbf{r}_{\mathbf{t}_{s}}=w_{s} \sum_{i} \mathbf{z}_{i}\left(x_{s} a_{i s}-b_{i s}\right)$. Applying (25) to (29) gives

$$
\begin{aligned}
g_{2}(\mathbf{x}, \mathbf{T}, \mathbf{W}) \leq & \sum_{s} \lambda_{\mathbf{t}_{s}} \mathbf{t}_{s}^{\prime} \mathbf{t}_{s}-2 \sum_{s} \lambda_{\mathbf{t}_{s}} \mathbf{t}_{s}^{\prime}\left(\mathbf{t}_{s 0}+\lambda_{\mathbf{t}_{s}}^{-1}\left[\mathbf{r}_{\mathbf{t}_{s}}-\mathbf{C}_{\mathbf{t}_{s}} \mathbf{t}_{s 0}\right]\right) \\
& +\sum_{s} \mathbf{t}_{s 0}^{\prime}\left(\lambda_{\mathbf{t}_{s}} \mathbf{I}-\mathbf{C}_{\mathbf{t}_{s}}\right) \mathbf{t}_{s 0}+k_{4} \\
= & \sum_{s} \lambda_{\mathbf{t}_{s}}-2 \sum_{s} \mathbf{t}_{s}^{\prime} \mathbf{p}_{s}+k_{6} \\
= & \sum_{s} \lambda_{\mathbf{t}_{s}}-2 \operatorname{tr} \mathbf{T}^{\prime} \mathbf{P}+k_{5}
\end{aligned}
$$

where $\lambda_{\mathbf{t}_{s}}$ is the largest eigenvalue of $\mathbf{C}_{\mathbf{t}_{s}}, \mathbf{p}_{s}=\lambda_{\mathbf{t}_{s}}\left(\mathbf{t}_{s 0}+\lambda_{\mathbf{t}_{s}}^{-1}\left[\mathbf{r}_{\mathbf{t}_{s}}-\mathbf{C}_{\mathbf{t}_{s}} \mathbf{t}_{s 0}\right]\right)$, $\mathbf{P}$ has columns $\mathbf{p}_{s}$, and $k_{5}=k_{4}+\sum_{s} \mathbf{t}_{s 0}^{\prime}\left(\lambda_{\mathbf{t}_{s}} \mathbf{I}-\mathbf{C}_{\mathbf{t}_{s}}\right) \mathbf{t}_{s 0}$. Note that the simplification $\lambda_{\mathbf{t}_{s}} \mathbf{t}_{s}^{\prime} \mathbf{t}_{s}=\lambda_{\mathbf{t}_{s}}$ makes use of the property of rotation matrices that the sum of squares of a column (or a row) equals one. Thus, (30) shows that $g_{2}(\mathbf{x}, \mathbf{T}, \mathbf{W})$ can be majorized by the linear function in $\mathbf{T},-2 \operatorname{tr} \mathbf{T}^{\prime} \mathbf{P}$ plus a constant. Let $\mathbf{P}=\mathbf{K} \mathbf{\Phi} \mathbf{L}^{\prime}$ be the singular value decomposition with $\mathbf{K}$ and $\mathbf{L}$ orthonormal and $\Phi$ diagonal with nonnegative diagonal elements. Then, the minimum of $-2 \operatorname{tr} \mathbf{T}^{\prime} \mathbf{P}$ is obtained for

$$
\mathbf{T}^{+}=\mathbf{K L}^{\prime}
$$

see, for example, Kiers and Groenen (1996).

This leads to the following algorithm. If dimension weighting is necessary, let $\delta_{\mathbf{W}}:=1$ otherwise set $\delta_{\mathbf{W}}:=0$. Similarly, set $\delta_{\mathbf{T}}:=1$ if rotations are necessary, otherwise set $\delta_{\mathbf{T}}:=0$.

1. Choose an initial $\mathbf{x}^{(0)}$. Set iteration counter $l:=0$ and set the convergence criterion $\epsilon_{\text {conv }}$ to a small positive constant. Set $\mathbf{W}^{(0)}=m^{1 / 2} \mathbf{I}$ and $\mathbf{T}^{(0)}=\mathbf{I}$.

2. $l:=l+1$. 
3. Calculate the update $\mathbf{x}^{+}$using formula (22) where $\mathbf{x}_{0}=\mathbf{x}^{(l-1)}, \mathbf{W}=$ $\mathbf{W}^{(l-1)}$, and $\mathbf{T}=\mathbf{T}^{(l-1)}$. Set $\mathbf{x}^{(l)}:=\mathbf{x}^{+}$.

4. If $\delta_{\mathbf{W}}=1$ calculate the update $\mathbf{W}^{+}$using formula (28) where $\mathbf{W}_{0}=$ $\mathbf{W}^{(l-1)}, \mathbf{x}=\mathbf{x}^{(l)}$, and $\mathbf{T}=\mathbf{T}^{(l-1)}$. Set $\mathbf{W}^{(l)}:=\mathbf{W}^{+}$.

5. If $\delta_{\mathbf{T}}=1$ calculate the update $\mathbf{T}^{+}$using formula (31) where $\mathbf{T}_{0}=$ $\mathbf{T}^{(l-1)}, \mathbf{x}=\mathbf{x}^{(l)}$, and $\mathbf{W}=\mathbf{W}^{(l)}$. Set $\mathbf{T}^{(l)}:=\mathbf{T}^{+}$.

6. Check for convergence: If $\left\|\mathbf{x}^{(l-1)}-\mathbf{x}^{(l)}\right\|>\epsilon_{\text {conv }}$ go to Step 2. 


\section{References}

Ahn, S. J., W. Rauh, H.-J. Warnecke. 2001. Least-squares orthogonal distances fitting of circle, sphere, ellipse, hyperbola, and parabola. Pattern Recogn. 34 2283-2303.

De Leeuw, J. 1994. Block relaxation algorithms in statistics. H. H. Bock, W. Lenski, M. M. Richter, eds. Information Systems and Data Analysis. Springer-Verlag, Berlin. 308-325.

De Leeuw, J., W. J. Heiser. 1980. Multidimensional scaling with restrictions on the configuration. P. R. Krishnaiah, eds. Multivariate Analysis V. North Holland, Amsterdam. 501-522.

Drezner, Z., S. Steiner, G.O. Wesolowsky. 2002. On the circle closest to a set of points Comput. Oper. Res. 29 637-650.

Eiselt, H. A., G. Laporte. 1995. Objectives in location problems. Z. Drezner, ed. Facility Location: A Survey of Applications and Methods. Springer, New York. 151-180.

Fonseca, M. J., J. A. Jorge. 2001. Experimental evaluation of an online scribble recognizer. Pattern Recogn. Lett. 22 1311-1319.

Groenen, P. J. F. 2002. Iterative majorization algorithms for minimizing loss functions in classification. Working paper presented at the 8th conference of the IFCS, Krakow, Poland, 2002.

Groenen, P. J. F., W. J. Heiser J. J. Meulman. 1999. Global optimization in least-squares multidimensional scaling by distance smoothing. J. Classif. $16225-254$.

Groenen, P. J. F., R. Mathar, W. J. Heiser. 1995. The majorization approach to multidimensional scaling for minkowski distances. J. Classif. $123-19$.

Groenen, P. J. F., B. J. van Os, J. J. Meulman. 2000. Optimal scaling by alternating length-constrained nonnegative least squares, with application to distance-based analysis. Psychometrika. 65 511-524.

Heiser, W. J. 1995. Convergent computation by iterative majorization: theory and applications in multidimensional data analysis. W. J. Krzanowski, ed. Recent Advances in Descriptive Multivariate Analysis. Oxford University Press, Oxford. 157-189.

Kiers, H. A. L. 2002. Setting up alternating least squares and iterative majorization algorithms for solving various matrix optimization problems. 
Comput. Stat. Data An. 41 157-170.

Kiers, H. A. L, P. J. F. Groenen. 1996. A monotonically convergent algorithm for orthogonal congruence rotation. Psychometrika 61 375-389.

Langbein, F. C., B. Mills, A. D. Marshall, R. R. Martin. 2001. Approximate geometric shapes. Int. J. Shape Model. 7 129-162.

Lange, K., D. R. Hunter, I. Yang. 2000. Optimization transfer using surrogate objective functions. J. Comput. Graph. Stat. 9 1-20.

Rorres, C., D. G. Romano. 1997. Finding the center of a circular starting line in an ancient greek stadium. SIAM Rev. 39 745-754.

Van-Ban, L., D. T. Lee. 1991. Out-of-roundness problem revisited. IEEE T. Pattern Anal. 13 217-223.

Van Deun, K., P. J. F. Groenen, W. J. Heiser, F. M. T. A. Busing, L. Delbeke. In Press. Interpreting degenerate solutions in unfolding by use of the vector model and the compensatory distance model. Psychometrika.

Yeralan, S., J. A. Ventura. 1998. Computerized roundness inspection. Int. J. Prod. Res. 26 1921-1935. 